\title{
Contribution
}

\section{à l'étude des oscillations périodiques des liquides pesants avec surface libre}

\section{A contribution to the study of periodic oscillations of ponderable liquids with a free surface}

PAR P. JOLAS,

INGÉNIEUR DE RECHERCHES A ÉLECTRICTTÉ DE FRANCE,

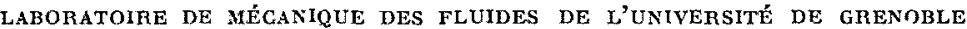

DEUXIEME PARTIE $(*)$

\section{EFFET D'UN SEUIL SUR LA PROPAGATION DE LA HOULE EN CANAL MESURE DU COEFFICIENT DE RÉFLEXION ET ANALYSE DE LA HOULE TRANSMISE}

\author{
SECOND SECTION (*) \\ THE EFFECT OF AN UNDERWATER BAR ON WAVE PROPAGATION IN A CANAL \\ MEASUREMENT OF THE REFLECTION COEFFICIENT AND ANALYSIS \\ OF THE TRANSMITTED WAVE
}

\begin{abstract}
Nous tentons de mettre en évidence expérimentalement un phénomène dont l'étude nous a été suggérée par le professeur J.J. Stoker : la houle incidente, après être passée sur un banc de sable immergé, sans déferler, engendre $\dot{a}$ l'aval de l'obstacle une série d'oscillations de courte longueur d'onde. Nous donnons les premiers résultats relatifs dà la mesure du coefficient de réflexion de l'obstacle et $\dot{a}$ l'analyse de la houle transmise.
\end{abstract}

\begin{abstract}
An attempt has been made to reproduce experimentally the phenomenon of an incident water wave which passes over an off shore underwater sand bar without breaking, and gives rise to a train of waves of short wave length downstream of the obstacle. The study of this phenomenon was suggested to us by Professor J.J. Stoker. Preliminary results are presented related to the measurement of the reflexion coefficient of the obstacle, and to the analysis of the transmitted wave.
\end{abstract}

On considère une houle plane se propageant dans un canal prismatique de section rectangulaire en profondeur constante, sauf dans une section où le canal présente un haut fond ou «seuil». Cet obstacle perturbe l'écoulement; la houle incidente est partiellement réfléchie vers l'amont et à l'aval existe une agitation résiduelle importante. En général, cette agitation est complexe et comporte une forte proportion d'harmoniques; ce n'est que si la profondeur relative au-dessus du seuil est suffisante que la houle transmise est pure. Après avoir rappelé le principe du calcul linéaire des coefficients de réflexion et de transmission de K. Takano, nous décrivons la manière dont nous procédons pour déterminer expérimentalement le coefficient de réflexion et analyser la houle transmise. Nous donnons ensuite les résultats de nos essais.

$\left(^{\star}\right)$ Voir la première partie : Sur la houle irrotationnelle en profondeur infinie, dans le $\mathrm{n}^{\circ} 5 / 1962$, page 635 .

$\left.{ }^{\star \star}\right)$ See the first section: About irrotational waves in infinite depth. No. $5 / 1962 ; p .635$. 
Cette étude dont nous donnons les premiers résultats est loin d'être terminée. C'est sur une suggestion du professeur J.J. Stoker que nous nous sommes intéressés à ce problème : sur certaines photos aériennes, on observe qu'une houle longue venant du large se transforme après être passée sans déferler sur un banc de sable ou un haut fond immergé en une série d'oscillations de courte longueur d'onde. N'ous avons essayé, en le schématisant, de mettre en évidence et d'analyser ce phénomène au Laboratoire.

\section{1. - CALCUL THEORIQUE DES COEFFICIENTS DE REFLEXION ET DE TRANSMISSION D'UN SEUIL}

Les résultats relatifs à la propagation de la houle en profondeur non constante sont obtenus à partir des équations linéarisées du mouvement. En effet, si, dans l'étude de la houle en profondeur constante, on est parvenu à mettre en évidence et à calculer des phénomènes non linéaires, le problème de la propagation de la houle en profondeur non constante est beaucoup moins classique et les calculs n'ont pu être développés jusqu'à présent que dans le cadre d'une théorie linéaire. On trouvera dans le livre Water waves de J.J. Stoker une revue des problèmes et des résultats obtenus récemment sur ce sujet, ainsi que dans le Handbuch der Physik [vol. IX, J.V. Wehausen, (1960)].

Soit un canal infini de profondeur uniforme, sauf dans une section où le profil du fond est donné par une loi de la forme $z=z(x)$, et une houle monopériodique plane se propageant de l' $\infty$ gauche vers la droite dans ce canal. A la rencontre de l'obstacle, cette houle donne naissance à une houle progressive transmise se propageant vers la droite et à une houle réfléchie se propageant vers la gauche.

On peut définir un coefficient de transmission et un coefficient de réflexion de l'obstacle comme il suit : on appellera coefficient de transmission le nombre complexe $\mathcal{C}_{t}$ dont le module $c_{b}$ est égal au rapport de l'amplitude de la houle transmise à l'amplitude de la houle incidente et dont l'argument est la différence entre la phase de l'onde transmise et celle de la houle incidente qui se serait propagée en l'absence d'obstacle. De même le module $c_{r}$ du coefficient de réflexion $\mathcal{C}_{r}$ est égal au rapport de l'amplitude de la houle réfléchie à celle de la houle incidente et son argument est la différence entre la phase de la houle réfléchie et celle de la houle incidente qui se serait réfléchie tótalement au droit du bord amont de l'obstacle.

Il est possible de traiter directement le problème dans le plan du mouvement pour des cas particuliers; plaque mince en profondeur infinie (Dean, 1945); plaque mince en profondeur finie (Dmitriev, 1954).

En transformant le domaine de l'écoulement en une bande, on parvient à démontrer un certain nombre de propriétés et de théorèmes d'existence. C'est ce qu'a fait Kreisel (1949), qui montre que le problème revient à résoudre une équation intégrale linéaire.

Tout récemment, en utilisant la méthode de Kreisel, T.F. Ogilvie (1960) est parvenu à démontrer l'existence et l'unicité du développement asymptotique du potentiel des vitesses moyennant certaines conditions sur la forme du fond; il traite numériquement le cas de la plaque mince en profondeur finie.

Mais il semble que pour certaines configurations, il soit plus commode d'opérer directement dans le plan du mouvement selon une méthode introduite par Apté (1957). Kenzo Takano, attaché de Recherche du C.N.R.S. au Laboratoire, a mis en équation et résolu numériquement le problème de cette façon pour un haut fond horizontal de longueur finie en profondeur finie. Nous rappelons ici le principe de son calcul.

Dans le plan du mouvement, on prend l'axe oz vertical ascendant suivant le bord amont de l'obstacle, $o x$ horizontal suivant le fond du canal dans le sens de la propagation des ondes incidentes. L'obstacle, de section rectangulaire, a une longueur $l$, une hauteur $h_{0}$, la profondeur d'eau dans le canal est $h$. On note T, $2 \pi / m, 2 \pi / \mu$ la période et les longueurs d'onde des houles en profondeur $h$ et $h-h_{0}$, respectivement.

On décompose le canal en trois domaines : amont, sur le seuil et aval: $D_{12}, D_{0}$ et $D_{3}$ définis respectivement par les inégalités:

$$
\begin{aligned}
& -\infty<x \leqslant 0 \quad 0 \leqslant 3 \leqslant h \quad\left(\mathrm{D}_{12}\right) \\
& 0<x<l \quad h_{0} \leqslant 3 \leqslant h \quad\left(\mathrm{D}_{0}\right) \\
& l \leqslant x<+\infty \quad 0 \leqslant 3 \leqslant h \quad\left(\mathrm{D}_{3}\right)
\end{aligned}
$$


On suppose le mouvement irrotationnel et on définit dans les domaines $D_{12}, D_{0}$ et $D_{3}$ respectivement, les potentiels $\Phi_{1}+\Phi_{12}, \Phi_{0}$ et $\Phi_{3}$ des houles incidente, réfléchie, de l'agitation sur le seưil et de la houle transmise sous la forme :

$$
\begin{aligned}
\Phi_{1} & =A_{1} \operatorname{ch} m z \exp (-i m x) \\
\Phi_{2} & =A_{2} \operatorname{ch} m z \exp (i m x)+\sum_{n=1}^{\infty} \mathrm{B}_{n} \cos \sigma_{n} z \exp \left(-\sigma_{n} x\right) \\
\Phi_{y_{1}} & =\mathrm{A}_{3} \operatorname{ch} m z \exp [-i m(x-l)]+\sum_{n=1}^{\infty} \mathrm{G}_{n} \cos \sigma_{n} z \exp \left[-\sigma_{n}(x-l)\right] \\
\Phi_{0} & =\mathrm{A}_{1} \operatorname{ch} \mu\left(z-h_{0}\right) \exp \left(i(\mu x)+\mathrm{A}_{5} \operatorname{ch} \mu\left(z-h_{0}\right) \exp (-i \mu x)\right. \\
& +\sum_{n=1}^{\infty}\left[\mathrm{C}_{n} \exp \left(-\lambda_{n} x\right)+\mathrm{D}_{n} \exp \left[-\lambda_{n}(x-l)\right] \cos \lambda_{n}(x-l)\right] \\
\mathrm{A}_{n}=a_{n} \exp \left[i\left(k t+\alpha_{n}\right)\right] & a_{1}=a_{1} \exp (i k t)
\end{aligned}
$$

les constantes $\sigma_{n}$ et $\lambda_{n}$ sont définies par les relations :

$$
\frac{4 \pi^{2}}{\mathrm{~T}^{2}}=k^{2}=-g \sigma_{n} \operatorname{tg} \sigma_{n} h=-g \lambda_{n} \operatorname{tg} \lambda_{n}\left(h-h_{0}\right)
$$

On montre, en effet, que, moyennant certaines hypothèses, remplies dans le cas considéré, $\Phi_{0}$, $\Phi_{2}$ et $\Phi_{3}$ admettent des développements du type précédent.

Les équations du phénomène étant linéaires et homogènes, on peut supprimer le facteur exp (ikt) et traiter les inconnues comme des constantes.

Les conditions aux limites sont les suivantes : pression constante à la surface libre, vitesse normale nulle aux parois, potentiel et gradient de potentiel continus aux frontières communes des domaines $D_{12}, D_{0}$ et $D_{3}$. On montre que ces conditions entraînent pour les inconnues complexes d'être solution d'un système linéaire infini.

Ce système est d'une forme beaucoup plus compliquée que celui obtenu pour un problème voisin : le passage en charge de la houle sous un obstacle : le problème du passage de la houle sous un obstacle peut être traité linéairement par des méthodes analogues à celles utilisées pour le passage de la houle sur un obstacle immergé; de nombreuses études théoriques et expérimentales ont déjà été publiées sur le sujet (Ursell, 1947, Macagno, 1954, Dean et Ursell, 1959, Takano, 1960). Elles montrent un accord satisfaisant entre les résultats expérimentaux et les prévisions théoriques, mais ce problème diffère essentiellement du nôtre; en effet, la présence de la surf́ace libre au-dessus de l'obstacle immergé est à l'origine de phénomènes nơn linéaires importants. C'est ce que nous montrons expérimentalement.

\section{Théorie linéaire approchée.}

Si la profondeur relative est suffisamment faible dans le canal et sur le seuil, on peut supposer la vitesse la même en tout point d'une section droite du canal ef faire l'hypothèse de. l'écoulement par tranche. Les équations du mouvement des ondes de gravité se simplifient considérablement et l'on peut en déduire une méthode générale d'étude de la propagation de la houle en présence d'obstacles de nature très diverse tels que : élargissement brusque, approfondissement brusque, filtres, etc. (Valembois, 1954). On trouve dans Lamb le calcul des coefficients de transmission et de réflexion pour un seuil semi-indéfini.

Pour un seuil de longueur finie, l'extension du calcul de Lamb a été faite par Jeffreys; il trouve les expressions explicites de $c_{t}$ et $c_{r}$ qui suivent:

$$
c_{i}=\frac{1}{\left[1+\frac{1}{4}\left(\frac{c_{0}}{c_{1}}-\frac{c_{1}}{c_{0}}\right)^{2} \sin ^{2} \frac{2 \pi l}{\lambda}\right]^{1 / 2}} \quad c_{r}=\frac{\frac{1}{2}\left[\frac{c_{0}}{c_{1}}-\frac{c_{1}}{c_{0}}\right] \sin \frac{2 \pi l}{\lambda}}{\left[1+\frac{1}{4}\left(\frac{c_{0}}{c_{1}}-\frac{c_{1}}{c_{0}}\right)^{2} \sin ^{2} \frac{2 \pi l}{\lambda}\right]^{1 / 2}}
$$

$c_{0}=$ célérité des ondes au-dessus du seuil;

$c_{1}=$ célérité des ondes dans le canal;

$l=$ longueur du seuil;

$\lambda=$ longueur d'onde de la houle incidente. 
D'après ces formules, on peut voir que les coefficients de réflexion et de transmission varient respectivement de 0 à une valeur maximale et de 1 à une valeur minimale qui dépendent du rapport de célérité des ondes longues dans le canal et sur le seuil.

\section{2. - DESCRIPTION DES ESSAIS}

\section{RESULTATS OBTENUS}

\section{a) Appareillage expérimental.}

Les essais ont été réalisés dans le canal à houle du Laboratoire de Mécanique des fluides de l'Université de Grenoble. Rappelons les caractéristiques de cette installation.

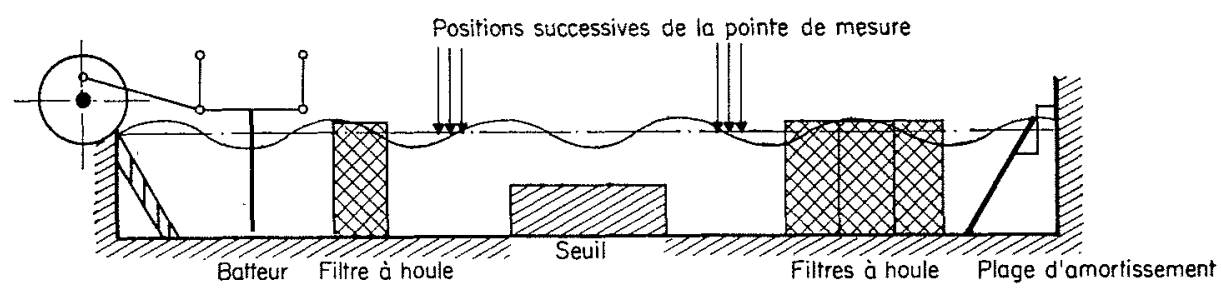

FIG. 1

Schéma de l'installation du canal à houle.

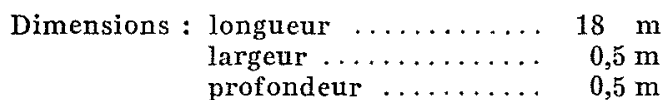

Le canal, de section $0,5 \times 0,5 \mathrm{~m}$ en ciment lissé intérieurement, long de $18 \mathrm{~m}$, est équipé d'un batteur trois barres. Ce batteur est entraîné par l'intermédiaire d'un système bielle-manivelle par ùn. groupe Ward-Leonard à régulation électronique; l'excentricité du système bielle-manivelle est réglable et permet de faire varier l'amplitude de l'onde émise par le batteur. La régulation dú mouvement permet d'en contrôler la période sur une gamme continue allant de 0,5 à $5 \mathrm{~s}$, et d'en garantir la constance à $0,001 \mathrm{~s}$ près. Le batteur trois barres a deux modes de fonctionnement possibles, en «translation» ou en «rotation». Nous l'avons utilisé en translation. Le volet utilisé était non étanche. A l'autre extrémité du canal, le dispositif d'amortissement de houle est constitué par une série de filtres et une plaque plane inclinée dont le bord inférieur repose sur le fond du canal et le hord supérieur affleure le plan d'eau au repos. Un mur vertical termine le canal. Le senil, immergé sensiblement au milieu du canal est en tôle d'aluminium; des joints en caoutchouc le stabilisent et assurent l'étanchéité.

L'instrument principal de nos mesures a été l'enregistreur de houle S. M (Santon, 1952). Le principe de cet appareil est d'enregistrer la durée d'immersion de l'extrémité d'une pointe en fonction de la profondeur à laquelle elle est immergée ou plus exactement les instants où la surface libre atteint l'extrémité de la pointe, soit à l'immersion, soit à l'émergence. L'appareil comporte essentiellement :

- un cylindre enregistreur recouvert de papier sensible télédeltos synchrone du volant d'entrainement du batteur et un chariot porte-stylet se déplaçant parallèlement à l'axe du cylindre avec une vitesse uniforme;

- une pointe de mesure montée sur chariot au-dessus du canal, pouvant se déplacer verticalement avec une vitesse uniforme, égale ou sous-multiple de celle du chariot porte-stylet.

On obtient ainsi, avec la pointe de mesure fixe, deux valeurs de la loi $z=z(t)$ de variation de cote de la surface libre en fonction du temps à une abscisse déterminée. En faisant varier lentenent la cote de l'extrémité de la pointe de mesure, on obtient, pourvu que le phénomène soit rigoureusement périodique; le profil de la loi $z=z(t)$ sur le papier d'enregistrement, en hachures régulières. 


\section{b) Méthodes de mesure.}

La mesure de l'amplitude des oscillations de la surface libre se fait par l'intermédiaire des enregistrements réalisés au $\mathrm{S}$. M. Grâce à la régulation électronique du mouvement d'entraînement du batteur, on peut regarder le phénomène comme périodique et effectuer au S. M. des enregistrements continus de la variation de cote de la surface libre en fonction du temps. On réalise ces enregistrements simultanément en deux abscisses. Par l'analyse harmonique de ces enregistrements, on connaît en amplitude et en phase, en deux abscisses données, les oscillations de la surface libre; il est

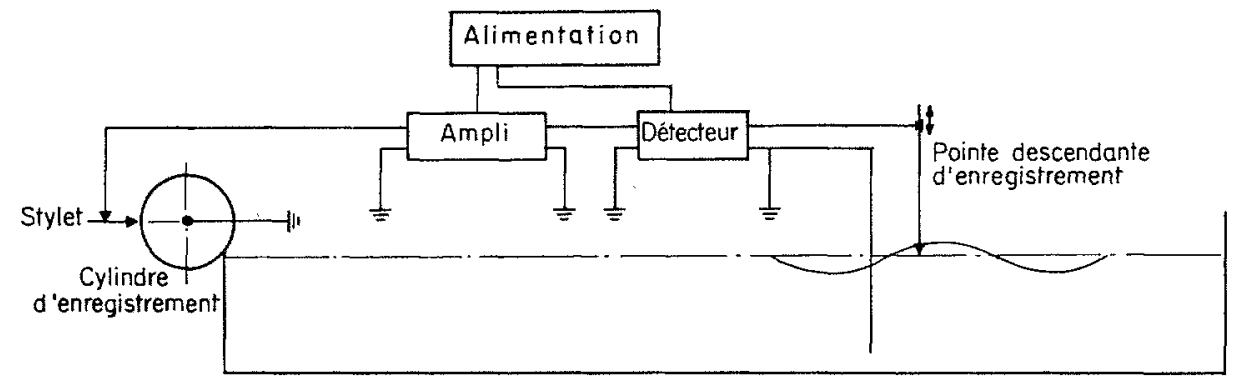

FIG. 2

Schéma électrique de l'enregistrement S.M.

alors facile, comme on le verra plus loin, d'en déduire le module et la phase relative des ondes incidentes et réfléchies fondamentales, puis harmoniques.

En fait, le phénomène étant périodique, on procède, non à des enregistrements simultanés, mais successifs, en déplaçant le chariot porte-pointe le long du canal successivement en plusieurs abscisses sans arrêter le système d'entraînement, de façon à conserver la même phase relative entre le cylindre enregistreur et le batteur.

On réalise ainsi en amont et en aval du seuil trois enregistrements : deux de mesure et un de contrôle, sur le même papier d'enregistrement.

La mesure de la période se fait sur un tour du volant d'entraînement du batteur, au moyen d'un périodemètre électronique constitué essentiellement d'un oscillateur donnant la base de temps et d'un compteur d'impulsions. Le signal de départ et de fin de comptage est donné par une cellule photoélectrique dont le pinceau lumineux est coupé à chaque tour du volant d'entraînement du batteur, par un bras fixé au volant. d'Airy :

La longueur d'onde est calculée à partir des valeurs mesurées de la période par la formule

avec

$$
k^{2}=m g \text { th } m h
$$

$$
k=\frac{2 \pi}{\mathrm{T}} \quad m=\frac{2 \pi}{\lambda}
$$

La profondeur d'eau $h$ est mesurée à la pointe limnimétrique.

\section{c) Dépouillement des enregistrements.}

L'analyse harmonique des profils enregistrés permet de connaitre en module et en phase, en un point du canal, les composantes harmoniques sinusoïdales du mouvement.

Cette analyse se fait par le calcul numérique approché, au moyen de grilles, à partir des données de la cote de la surface libre en « 24 points , c'est-à-dire en 24 instants successifs et régulièrement espacés sur une période. Les grilles que nous utilisons, adaptées au calcul à la machine à calculer électrique, permettent de faire rapidement l'analyse jusqu'au sixième harmonique.

Nous avons pu aussi nous servir d'un analyseur mécanique de Ott. Le principe de cet appareil est de planimétrer les intégrales de définition des coefficients de Fourier :

$$
a_{n}=\frac{1}{2 \mathrm{~T}} \int_{0}^{\mathrm{T}} f(t) \cos \frac{2 \pi n t}{\mathrm{~T}} d t \quad b_{n}=\frac{1}{2 \mathrm{~T}} \int_{0}^{\mathrm{T}} f(t) \sin \frac{2 \pi n t}{\mathrm{~T}} d t
$$

en parcourant la courbe à étudier avec une pointe suiveuse. On y parvient grâce à l'interposition, entre la pointe suiveuse et la pointe du planimètre, d'un «guide analyseur ». Les possibilités sont 
étendues, puisqu'on peut faire l'analyse jusqu'à l'harmonique 33 et, lorsqu'on se limite au fondamental, on réalise une économie de temps appréciable par rapport au «calcul en 24 points». Cependant la précision est légèrement inférieure à celle de l'analyse numérique.

Le dépouillement proprement dit procède par approximations successives. Nous négligeons en première approximation les termes non linéarisés étudiés par Biesel (1952), Kravtchenko et Santon (1957), Daubert (1958), et nous admettons que les oscillations de la surface libre sont représentables par un nombre complexe de la forme:

$$
z(t)=A \exp \left[i\left(\frac{2 \pi t}{\mathrm{~T}}+\varphi\right)\right]=z(t)
$$

où -A est l'amplitude et $\varrho$ la phase de l'oscillation.

Une onde progressive se propageant dans le sens des $x$ croissants est représentée symboliquement par le nombre complexe :

$$
A \exp \left[i\left(\frac{2 \pi t}{\mathrm{~T}}-\frac{2 \pi x}{\lambda}+\alpha\right)\right]=z \exp [-i \beta]
$$

où $\lambda$ est la longueur d'onde correspondant à la période $T$ d'après la formule d'Airy. Il suffit de changer le signe de $\lambda$ pour avoir une représentation complexe de l'onde réfléchie. La correction de longueur d'onde due à la cambrure est inférieure à la précision de nos expériences, de telle sorte que l'approximation d'Airy, qui est d'ailleurs très bonne, est suffisante.

Les résultats de l'analyse harmonique des profils temporels enregistrés simultanément en deux abscisses déterminées fournissent deux relations qui permettent de déterminer les quantités complexes représentant l'onde incidente et l'onde réfléchie à une abscisse donnée. En effet, si on note $z$ l'onde incidente et $z^{\prime}$ l'onde réfléchie à la première abscisse, $B_{1}$ et $B_{2}$ les résultats de l'analyse harmonique à la première et à la deuxième abscisse, on a :

$$
\begin{aligned}
& z+z^{\prime}=\mathrm{B}_{\mathrm{t}} \\
& z \exp \left[i 2 \pi \frac{\left(x_{2}-x_{1}\right)}{\lambda}\right]+z^{\prime} \exp \left[-i 2 \pi \frac{\left(x_{2}-x_{1}\right)}{\lambda}\right]=\mathrm{B}_{2} \\
& z=\frac{-i}{2 \sin \left[2 \pi\left(x_{2}-x_{1}\right) / \lambda\right]}\left\{\mathrm{B}_{2}-\mathrm{B}_{1} \exp \left[-i \frac{2 \pi\left(x_{2}-x_{1}\right)}{\lambda}\right]\right\} \\
& z^{\prime}=\frac{-i}{2 \sin \left[2 \pi\left(x_{2}-x_{1}\right) / \lambda\right]}\left\{\mathrm{B}_{1} \exp \left[\frac{-i 2 \pi\left(x_{2}-x_{1}\right)}{\lambda}\right]-\mathrm{B}_{2}\right\}
\end{aligned}
$$

En fait, on procède à plusieurs enregistrements si multanés; voici, plus détaillée que dans Marcou (1957), la méthode employée :

Soient pour plus de généralité :

$A_{n}$ l'amplitude de l'harmonique incident d'ordre $n$;

$A^{\prime}{ }_{n}$ l'amplitude de l'harmonique réfléchi d'ordre $n$;

$n$ le rang de l'harmonique de période $\mathrm{T} / n$;

$\lambda_{n}$ la longueur d'onde de l'harmonique $n$ supposé connue (pratiquement calculée par la formule d'Airy et en négligeant la correction de cambrure);

$p$ le $\mathrm{n}^{\circ}$ de l'enregistrement $(p=1,2,3 \ldots \mathrm{P})$; les enregistrements du profil sont faits en un nombre $\mathrm{P}$ d'abscisses numérotées dans le sens de la propagation de la houle incidente;

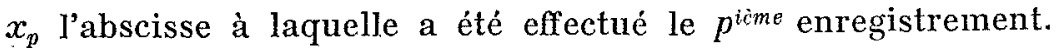

Rappelons que l'on note respectivement $z_{n p}$ et $z_{n p}^{\prime}$ les nombres complexes caractérisant les houles incidentes et réfléchies :

$$
\begin{gathered}
z_{n p}=\mathrm{A}_{n} \exp \left[i\left(\frac{2 \pi t}{\mathrm{~T}_{n}}-\frac{2 \pi x_{p}}{\lambda_{n}}+\rho_{n}\right)\right]=z_{n} \exp \left[i \frac{2 \pi x_{p}}{\lambda_{n}}\right] \\
z_{n p}^{\prime}=\mathrm{A}_{n} \exp \left[i\left(\frac{2 \pi t}{\mathrm{~T}_{n}}+\frac{2 \pi x_{p}}{\lambda_{n}}+\rho_{n}^{\prime}\right)\right]=z_{n}^{\prime} \exp \left[-i \frac{2 \pi x_{p}}{\lambda_{n}}\right]
\end{gathered}
$$

On observera que toutes les quantités que nous avons à considérer contiennent le facteur $\exp \left[i\left(2 \pi n t / \mathrm{T}_{n}\right)\right]$. 
On pourra donc toujours supprimer cette fonction sinusoïdale de $t$ et traiter $z_{n}$ et $z_{n}^{\prime}$ comme des quantités complexes constantes.

L'analyse harmonique donne directement les quantités :

$$
\mathrm{B}_{n p}=\mathrm{A}_{n p} \exp \left[i\left(\frac{2 \pi t}{\mathrm{~T}_{n}}+\varphi_{n p}\right)\right]
$$

Or l'oscillation résultante en $x_{p}$ est représentée par $z_{n p}+z_{n p}^{\prime}$. A chaque abscisse on a donc la relation linéaire :

$$
z_{n} \exp \left[\left(\frac{2 \pi x_{p}}{\lambda_{n}}\right) i\right]+z_{n}^{\prime} \exp \left[-\left(\frac{2 \pi x_{p}}{\lambda_{n}}\right) i\right]=\mathrm{B}_{n p}
$$

Comme on l'a déjà souligné, deux équations du type ci-dessus suffisent à déterminer les quantités complexes $z_{n}$ et $z_{n}^{\prime}$.

Si nous disposons de $\mathrm{P}$ enregistrements simultanés effectués en $\mathrm{P}$ abscisses différentes, on a $\mathrm{P}$ équations de la forme :

$$
z_{n} \exp \left[\left(\frac{2 \pi x_{p}}{\lambda_{n}}\right) i\right]+z_{n}^{\prime} \exp \left[-\left(\frac{2 \pi x_{p}}{\lambda_{n}}\right) i\right]-\mathrm{B}_{n p}=\varepsilon_{n p}
$$

L'application de la méthode classique des moindres carrés consiste à rendre minimale la somme

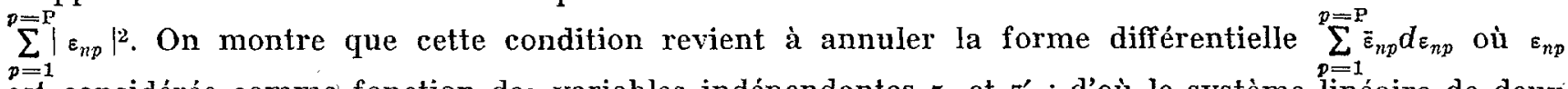

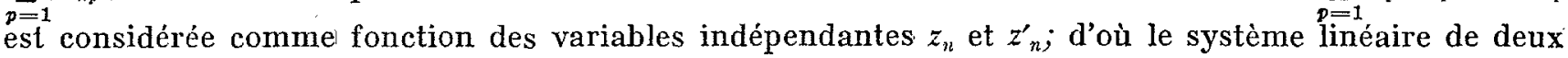
équations à deux inconnues :

$$
\begin{gathered}
\sum_{p=1}^{p=\mathrm{P} z_{n}} \exp \left[\left(\frac{4 \pi x_{p}}{\lambda_{n}}\right) i\right]+\mathrm{P} z_{n}^{\prime}=\sum_{p=1}^{p} \sum_{p}^{\mathrm{P}} \mathrm{B}_{p} \exp \left[\left(\frac{2 \pi x_{p_{-}}}{\lambda_{n}}\right) i\right] \\
\mathrm{P} z_{n}+\sum_{p=1}^{p=} z_{n}^{\prime} \exp \left[-\left(\frac{4 \pi x_{p}}{\lambda_{n}}\right) i\right]=\sum_{p=1}^{p} \overline{\bar{p}}^{\mathrm{P}} \mathrm{B}_{p} \exp \left[-\left(\frac{2 \pi x_{p}}{\lambda_{n}}\right) i\right]
\end{gathered}
$$

dont la solution s'écrit :

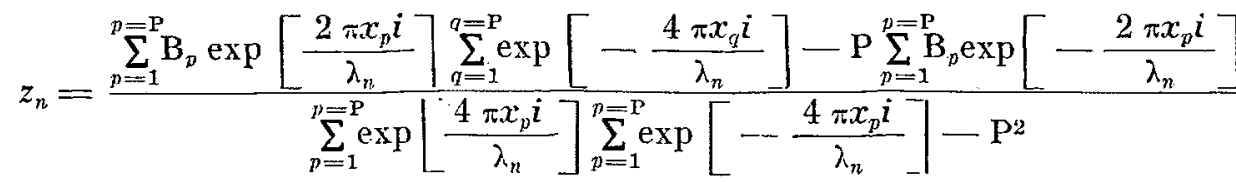

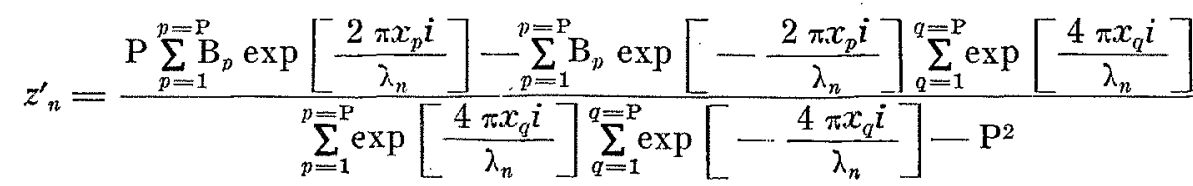

En pratique, on se limite à faire trois enregistrements simultanés.

Explicitons les calculs dans ce cas particulier : on pose :

$$
\begin{array}{ll}
\alpha=\frac{2 \pi x_{1}}{\lambda_{n}}=0 & \mathrm{~B}_{a}=\mathrm{B}_{1} \\
\beta=\frac{2 \pi x_{2}}{\lambda_{n}} & \mathrm{~B}_{\beta}=\mathrm{B}_{2} \\
\gamma=\frac{2 \pi x_{3}}{\lambda_{n}} & \mathrm{~B}_{\gamma}=\mathrm{B}_{3}
\end{array}
$$

On a alors aux trois ạbscisses les relations suivantes:

$$
\mid \begin{aligned}
& z_{n}+z_{n}^{\prime}-\mathrm{B}_{a}=\varepsilon_{\alpha} \\
& z_{n} \exp [\beta i]+z^{\prime}{ }_{n} \exp [-\beta i]-\mathrm{B}_{\beta}=\varepsilon_{\beta} \\
& z_{n} \exp [\gamma i]+z_{n}^{\prime} \exp [-\gamma i]-\mathrm{B}_{\gamma}=\varepsilon_{\gamma}
\end{aligned}
$$




$$
\begin{gathered}
\left\{\begin{array}{l}
d \varepsilon_{\alpha}=d z_{n}+d z_{n}^{\prime} \\
d \varepsilon_{\beta}=\exp [\beta i] d z_{n}+\exp [-\beta i] d z_{n}^{\prime} \\
d \varepsilon_{\gamma}=\exp [\gamma i] d z_{n}+\exp [-\gamma i] d z^{\prime}{ }_{n}
\end{array}\right. \\
\varepsilon_{a} d \varepsilon_{\alpha}+\varepsilon_{\beta} d \varepsilon_{\beta}+\varepsilon_{\gamma} d \varepsilon_{\gamma}=d z_{n}\left[\begin{array}{c}
z_{z_{k}}+z_{n}^{\prime}-\mathrm{B}_{\alpha}+\exp (\beta i)\left[z_{n} \exp (\beta i)+z_{n}^{\prime} \exp (-\beta i)-\mathrm{B}_{\beta}\right] \\
+\exp (\gamma i)\left[z_{n} \exp (\gamma i)+z_{n}^{\prime} \exp (-\gamma i)-\mathrm{B}_{\gamma}\right.
\end{array}\right]
\end{gathered}
$$

d'où

$$
\begin{gathered}
z_{n}[1+\exp [2 \beta i]+\exp (2 \gamma i)]+3 z_{n}^{\prime}=\mathrm{B}_{\alpha}+\mathrm{B}_{\beta} \exp [\beta i]+\mathrm{B}_{\gamma} \exp [\gamma i] \\
3 z_{n}+z_{n}^{\prime}[1+\exp [-2 \beta i]+\exp [-2 \gamma i]]=\mathrm{B}_{\alpha}+\mathrm{B}_{\beta} \exp [-\beta i]+\mathrm{B}_{\gamma} \exp [-\gamma i]
\end{gathered}
$$

la solution s'écrit :

avec :

$$
z_{n}=\sum_{q=1}^{3} b_{q} B_{q} \quad z_{n}^{\prime}=\sum_{q=1}^{3} \delta_{q} B_{q}
$$

$$
\begin{gathered}
b_{\alpha}=\frac{\sin ^{2} \beta+\sin ^{2} \gamma+i[\sin \beta \cos \beta+\sin \gamma \cos \gamma]}{2\left[\sin ^{2} \beta+\sin ^{2} \gamma+\sin ^{2}(\gamma-\beta)\right]} \\
b_{\beta}=\frac{\sin (\gamma-\beta) \sin \gamma+i[\sin (\gamma-\beta) \cos \beta-\sin \beta]}{2\left[\sin ^{2} \beta+\sin ^{2} \gamma+\sin ^{2}(\gamma-\beta)\right]} \\
b_{\gamma}=\frac{-\sin (\gamma-\beta) \sin \beta+i[-\sin (\gamma-\beta) \cos \gamma-\sin \gamma]}{2\left[\sin ^{2} \beta+\sin ^{2} \gamma+\sin ^{2}(\gamma-\beta)\right]}
\end{gathered}
$$

On calcule ensuite les écarts $\varepsilon_{n p}$, et l'écart quadratique moyen permet de voir avec quelle précision la théorie linéaire représente le phénomène.

La seconde approximation consiste à prendre en compte les termes du second ordre de la houle complexe. On reprend le calcul précédent, mais avec des valeurs de $\mathbf{B}_{q}$ corrigées par les termes non linéaires suivants : dans le calcul du fondamental, on tient compte des interactions entre les harmoniques 1 et $2: \gamma_{2} z_{2} \bar{z}_{1}$ et 2 et $3: \gamma_{23} z_{3} \bar{z}_{2}$; dans le calcul de l'harmonique $2:$ du terme du second ordre de la houle pure $M z_{1}{ }^{2}$ et du terme d'interaction $\gamma_{31} z_{3} \bar{z}_{1}$ entre les harmoniques 3 et 1; dans le calcul de l'harmonique 3 , du terme d'interaction entre les harmoniques 1 et $2 \eta_{12} z_{1} z_{2}$. Les termes d'interaction faisant intervenir lés ondes réfléchies et des harmoniques d'ordre supérieur à 3 ne sont pas pris en compte, car leur ordre de grandeur est trop faible. Des abaques, tracés d'après les formules de Biesel (1952) donnent les valeurs des coefficients des termes d'interaction.

\section{d) Détermination expérimentale des coefficients de transmission et de réflexion.}

Après analyse harmonique des enregistrements de houle amont et aval, soient $A_{1}$ l'amplitude du fondamental progressif amont, $A_{2}$ celle du réfléchi, $A_{3}$ celle du fondamental progressif aval. On a les relations:

$$
c_{t}=\frac{\left|z_{3}\right|}{\left|z_{1}\right|}=\frac{\mathrm{A}_{3}}{\mathrm{~A}_{1}} \quad c_{r}=\frac{\left|z_{2}\right|}{\left|z_{1}\right|}=\frac{\mathrm{A}_{2}}{\mathrm{~A}_{1}}
$$

Comme le canal n'est pas indéfini, on doit tenir compte de l'onde réfléchie par la plage : soient ${ }_{a m} x_{1}$ et ${ }_{a v} x_{1}$ les abscisses d'enregistrement amont et aval, et $\mathcal{C}_{t}=\left(\bar{z}_{1} z_{2} /\left|z_{1}\right|^{2}\right)$ le coefficient complexe de transmission : on a :

$$
c_{r}=\frac{\left|z_{2}-z_{4} \mathcal{C}_{t} \exp \left[2 \pi\left({ }_{a v} x_{1}-{ }_{a m} x_{1}\right)\right] / \lambda_{1}\right|}{\left|z_{1}\right|}
$$


Il y a une correction analogue pour le calcul de $c_{t}$ et on peut procéder par approximations successives; mais dans le cas. d'un obstacle immergé où $c_{t}$ est grand et $c_{r}$ petit, cette correction est négligeable.

On montre que ce schéma de calcul donne bien $c_{r}$ et $c_{t}$ du seuil, quel que soit le nombrè de réflexions successives de l'onde fondamentale aux extrémités du canal, soit sur le batteur, soit sur la plage d'amortissement. Il suffit de connaître globalement les ondes progressives et réfléchies à l'amont et à l'aval du seuil pour calculer les coefficients $c_{r}$ et $c_{t}$. Il faut prendre la précaution, avant de procéder à des enregistrements, d'attendre que le régime du canal soit « établi ».

Ce procédé est nouveau pour déterminer des coefficients de réflexion et semble plus précis que celui qui consiste à mesurer les amplitudes aux nœuds et aux ventres du clapotis partiel. Il permet une détermination séparée et précise pour le fondamental et les harmoniques. Signalons à ce sujet une méthode très intéressante basée sur une propriété non linéaire du clapotis partiel (Cooper and Longuet-Higgins, 1951) : celle d'avoir, dans l'expression de la pression, des termes du second ordre dont l'amplitude ne dépend pas de la profondeur : on a ainsi, au fond, des pulsations de fréquence double dont l'amplitude est proportionnelle à l'amplitude de l'onde incidente et à celle de l'onde réfléchie. On peut, par des mesures successives de la réflexion sur l'obstacle et sur un mur vertical, déduire facilement le coefficient de réflexion de l'obstacle, connaissant celui du mur vertical (Domzig, 1955).

Ơn a comparé, pour quatre périodes, les coefficients calculés par la théorie exacte et par la théorie linéarisée, aux valeurs expérimentales. Les conditions des essais étaient les suivantes :

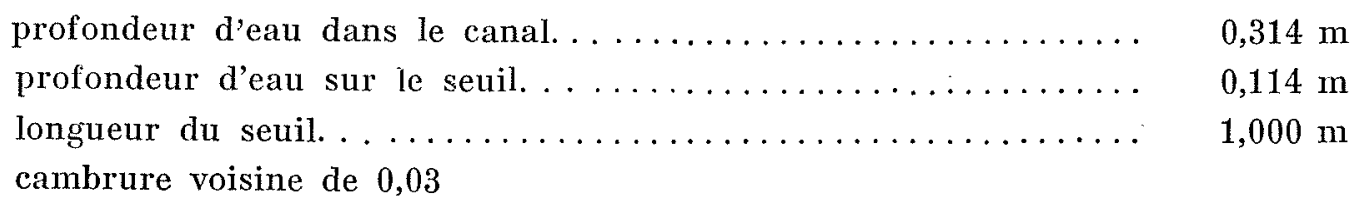

\begin{tabular}{|c|c|c|c|c|c|c|}
\hline \multirow{2}{*}{$\begin{array}{l}\text { Période } \\
\text { (s) }\end{array}$} & \multicolumn{2}{|c|}{ Valeurs expérimentales } & \multicolumn{2}{|c|}{$\begin{array}{l}\text { Théorie linéaire exacte } \\
\qquad(n=1)\end{array}$} & \multicolumn{2}{|c|}{ Théorie linéarisée } \\
\hline & $\mathrm{C}_{r}$ & $\mathrm{C}_{t}$ & $\mathrm{C}_{r}$ & $\mathrm{C}_{t}$ & $\mathrm{C}_{r}$ & $\mathrm{C}_{t}$ \\
\hline 0,8161 & 0,04 & 0,91 & 0,09 & 0,99 & 0,47 & 0,88 \\
\hline 0,9105 & 0,17 & 0,87 & 0,21 & 0,98 & 0,56 & 0,82 \\
\hline 1,0055 & 0,22 & 0,84 & 0,28 & & 0,17 & 0,98 \\
\hline 1,1024 & 0,11 & 0,85 & 0,17 & 0,96 & 0,27 & 0,96 \\
\hline
\end{tabular}

Il $\mathrm{y}$ a assez bon accord entre les valeurs de la théorie exacte et les valeurs expérimentales $( \pm 6 \%)$, pour le coefficient de réflexion. Pour le coefficient de transmission, la différence est toujours grande : cette différence peut, en partie, être expliquée par les pertes d'énergies, car l'écoulement est loin d'être irrotationnel au voisinage de l'obstacle; d'autre part, la théorie linéaire exacte, a priori ne tient pas compte des harmoniques; alors que $c_{r}{ }^{2}+c_{t}{ }^{2}=1$ théoriquement, on trouve avec les valeurs expérimentales, un déficit d'au moins $20 \%$. La précision relative des mesures est de 0,05 . On doit pouvoir obtenir des résultats plus précis grâce à des enregistrements effectués plus loin des bords amont et aval du seuil. Nous étions limité par la faible longueur du canal.

Un phénomène négligé par la théorie linéaire est la variation du coefficient $c_{r}$ avec l'amplitude : on constate que le coefficient de réflexion augmente avec la cambrure (il augmente de $10 \%$ cnviron quand la cambrure passe de 0,02 à 0,04 ) pour diminuer ensuite vraisemblablement; mais la naissance du déferlement sur le seuil ne nous a pas permis des mesures précises pour de trop fortes cambrures. 


\section{e) Analyse de la houle à l'aval du seuil.}

On observe en général, à l'aval du seuil, une agitation complexe. Le profil de la surface libre n'est plus indéformable comme celui d'une houle pure, mais celui d'une série d'oscillations se propageant à des vitesses différentes. L'enregistrement du profil temporel de la surface libre montre cependant que les oscillations sont périodiques de période fondamentale, celle de la houle incidente. On a donc une superposition d'ondes harmoniques.

Un enregistrement effectué pour une période de $1,5080 \mathrm{~s}$ (ce qui correspond à une profondeur relative de 0,07 sur le seuil) a donné les résultats suivants : ayant fait un dépouillement linéaire, suivant la méthode indiquée au chapitre $(b)$, des enregistrements amont et aval, on constate que les harmoniques 1,2 et 3 , qui ont à l'aval une amplitude importante, se propagent sensiblement à leur célérité propre (célérité d'Airy)). Nous n'avons fait qu'une évaluation des harmoniques 4, 5, 6 : pour connaitre leur ordre de grandeur, nous prenons la moyenne des amplitudes aux trois abscisses d'enregistrement. On trouve à l'amont les résultats suivants :

\begin{tabular}{|c|c|c|c|}
\hline Ordre de l'harmonique & $\begin{array}{c}\text { Amplitude de l'incident } \\
(\text { en } \mathrm{cm})\end{array}$ & $\begin{array}{l}\text { Amplitude du réfléchi } \\
\qquad(\mathrm{en} \mathrm{cm})\end{array}$ & $\begin{array}{c}\text { Ecart quadratique moyen } \\
(\mathrm{en} \mathrm{cm})\end{array}$ \\
\hline 1 & 1,53 & 0,32 & 0,02 \\
\hline 2 & 0,10 & 0,08 & 0,06 \\
\hline 3 & 0,02 & & \\
\hline
\end{tabular}

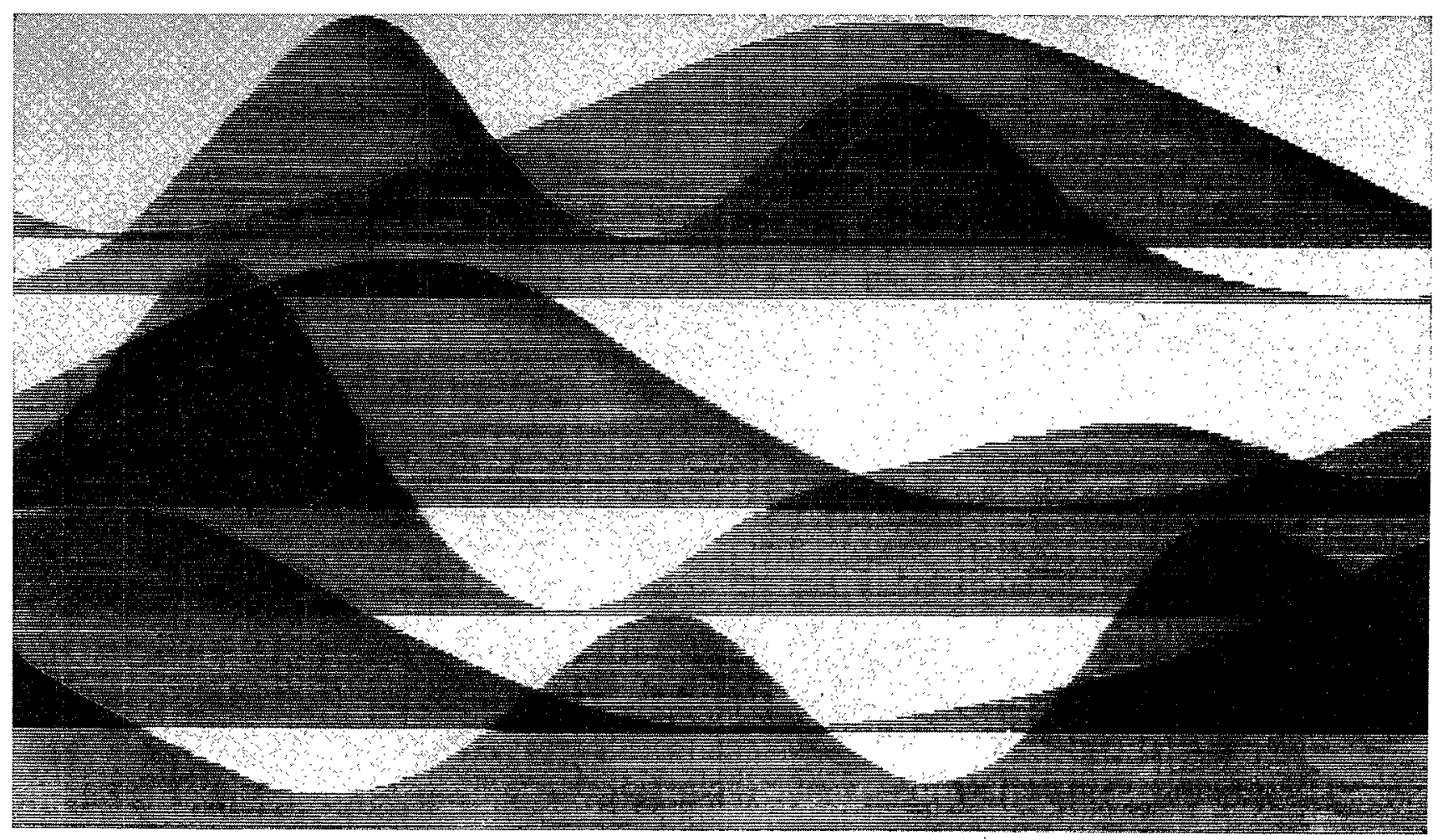

Fig. 3

Exemple d'enregistrement. 
et à l'aval :

\begin{tabular}{|c|c|c|c|}
\hline Ordre de l'harmonique & $\begin{array}{c}\text { Amplitude de l'incident } \\
\text { (en cm) }\end{array}$ & $\begin{array}{c}\text { Amplitude du réfléchi } \\
\text { (en cm) }\end{array}$ & $\begin{array}{c}\text { Ecart quadratique moyen } \\
\text { (en cm) }\end{array}$ \\
\hline 1 & 1,02 & 0,10 & 0,04 \\
2 & 1,20 & 0,04 & 0,05 \\
3 & 0,31 & 0,05 & 0,05 \\
4 & 0,07 & & \\
6 & 0,06 & & \\
\end{tabular}

Une série d'essais nous a ensuite montré que les harmoniques apparaissent à l'aval quand la profondeur relative au-dessus du seuil est inférieure à 0,15 environ. Mais c'est une limite approximative, car si le paramètre prépondérant dans cette étude est la profondeur relative au-dessus du seuil, la cambrure de la houle incidente et la longueur relative du seuil sont aussi des paramètres importants.

Nous donnons ci-après à titre indicatif les résultats d'une série d'essais.

\begin{tabular}{|c|c|c|c|c|c|c|c|c|}
\hline \multirow{2}{*}{$\begin{array}{l}\text { Période } \\
\text { fondamentale } \\
\text { (en s) }\end{array}$} & \multirow{2}{*}{$\begin{array}{l}\text { Longueur } \\
\text { d'onde } \\
\text { (en } \mathrm{cm} \text { ) }\end{array}$} & \multirow{2}{*}{$\begin{array}{l}\text { Profondeur } \\
\text { relative } \\
\text { au-dessus } \\
\text { du seuil }\end{array}$} & \multicolumn{6}{|c|}{ Amplitude des harmoniques aval (en $\mathrm{cm}$ ) } \\
\hline & & & 1 & 2 & 3 & 4 & 5 & 6 \\
\hline $0,816.1$ & 100 & 0,14 & 1,73 & 0,09 & & & & \\
\hline 0,9202 & 120 & 0,13 & 1,65 & 0,26 & 0,08 & & & \\
\hline 1,0055 & 140 & 0,11 & 1,54 & 0,39 & 0,08 & & & \\
\hline 1,1024 & 160 & 0,10 & 1,56 & 0,37 & 0,06 & & & \\
\hline 1,2014 & 180 & 0,09 & 1,36 & 0,64 & 0,17 & & & \\
\hline 1,3020 & 200 & 0,08 & 1,23 & 0,93 & 0,21 & 0,03 & 0,04 & 0,02 \\
\hline 1,4044 & 220 & 0,08 & 0,98 & 1,08 & 0,23 & 0,07 & 0,07 & 0,03 \\
\hline 1,5080 & 240 & 0,07 & 1,02 & 1,20 & 0,31 & 0,07 & 0,06 & 0,02 \\
\hline
\end{tabular}

Au fur et à mesure que la profondeur relative au-dessus du seuil diminue, on observe que le maximum du spectre des amplitudes de la houle en aval du seuil se déplace vers les harmoniques élevés.

Remarque. - L'analyse de la houle, au-dessus du seuil, est rendue difficile par la faible longueur de celui-ci. Au cours de sa progression sur le seuil, la houle se modifie rapidement. Nous avons fait des enregistrements du profil temporel de la surface libre au droit du bord amont, au milieu et au droit du bord aval du seuil. Les profils obtenus au milieu et au bord aval sont sensiblement ceux d'une houle cnoïdale.

On sait en effet que lorsque la profondeur d'eau est inférieure au $1 / 10^{\text {e }}$ de la longueur d'onde, environ, la théorie classique de Stokes n'est plus susceptible de représenter correctement les phénomènes (Keulegan 1950, De, 1955). La théorie de la houle cnoïdale, apparaît être beaucoup plus satisfaisante (Taylor, 1955, Weigel, 1959); nous avons donc de fortes raisons de penser qu'après un parcours assez court en profondeur très faible, la houle ait ensuite un profil indéformable qui soit celui d'une houle cnoïdale. Nous n'avons pas tenté d'étudier la transformation de la houle au'-dessus du seuil, car cela demande une mise au point de l'appareillage expérimental.

Au point de vue théorique, rappelons que l'existence de la solution du problème des ondes du type cnoïdal a été démontrée par Littman (1957), par une méthode voisine de celle utilisée par Friedrichs et Hyers pour démontrer l'existence de l'onde solitaire. 
L'analyse harmonique du profil enregistré au droit du bord aval du seuil nous a donné les résultats suivants :

\begin{tabular}{|c|c|c|c|}
\hline Ordre de l'harmonique & $\begin{array}{c}\text { Amplitude } \\
(\mathrm{en} \mathrm{cm})\end{array}$ & Ordre de l'harmonique & $\begin{array}{c}\text { Amplitude } \\
\text { (en cm) }\end{array}$ \\
\hline 1 & 1,09 & 4 & 0,28 \\
1,22 & 5 & 6 & 0,15 \\
0,59 & 0,06 & 5 \\
\hline
\end{tabular}

On notera la similitude entre spectres d'amplitude de la houle sur le bord amont du seuil et en aval (où les harmoniques se propagent alors, rappelons-le, à leur célérité propre).

\section{RĒFÉRENCES}

APTÉ (A.). - Recherches théoriques et expérimentales sur les mouvements des liquides pesants à surface libre. Thèse, Grenoble, 1957. Publications Scientifiques et Techniques du Ministère de l'Air.

Biesel ( $F$.): - Equations générales de la houle au second ordre de la houle irrégulière. La Houille Blanche, 1952, (372-376).

Cooper (R. I. B.) and Longuet-Higgins (M. S.). - An experimental study of the pressure variation in standing water waves. Proc. of the Roy. Soc., London, 1951, (424-435).

Daubert (A.). - Calcul de la houle complexe au troisième ordre. Thèse, Grenoble, 1958 (à paraître dans la Collection Scientifique et Technique du Ministère de l'Air).

$\mathrm{DE}$ (S. C.). - Contribution to the theory of Stokes's waves. Proc. Camb. Phil. Soc., 1955, (713-736).

DeAN (W.R.). - On the reflexion of surface waves by a submerged plane barrier. Proc. Camb. Phil. Soc., $1945,(232-238)$.

Dean (W.R.) et UrSell (F.). - Interaction of a fixed semi-immersed circular cylinder with a train of surface waves. Massachussetts Institute of Technology, Technical Report $\mathrm{n}^{\circ}$ 37, 1959.

Dmitriey (A.A.). - Passage d'ondes planes au-dessus d'un mur noyé. Comptes rendus de l'Académie des Sciences d'U.R.S.S., 1954, (981-984), (en russe).

Domzic (H.). - Wellendruck und Druckerzengender Seegang. Mitt. Hann. Versuchsanst. für Grund- und Wasserbau, 1955, Heft 8.

Jefrreys (H.). - Wave Report $n^{\circ} 3$, Ministry of Supply, 1944.

Keulegan (G. H.). - Wave motion. Proc. IV Conf. Engineering Hydraulics, edited by H. Rouse, N.Y., 1950.

Kravtchenko (J.) et Santon (L.). - Nouvelles recherches sur la houle de laboratoire. Communication A.I.R.H., Lisbonne, $1957\left(\mathrm{D}_{2}\right)$.

Krerser (G.). - Surface waves. Quarterly of Applied Math,. 1949, (21-44).
LaMb (H.). - Hydrodynamics, 1879, (262-263).

Litrman (W.). - On the existence of periodic waves near eritical speed. Comm. on Pure and Applied Mathematics, 1957, (241-260).

Magagno (F.O.). - Houle dans un canal présentant un obstacle en charge. Thèse, Grenoble, 1954, publiée dans La Houille Blanche, $\mathrm{n}^{\circ} 1,1954$.

Marcou (C.). - Plage d'amortissement pour canal à houle. Proc. V. Conf. Coastal Engineering, Grenoble, 1952, (258-267).

Marcou (C.). - Mesure précise de la houle de laboratoire. Communication A.I.R.H., Lisbonne, $1957\left(\mathrm{D}_{1}\right)$.

OGILvis (T.F.). - Propagation of waves over an obstacle in water of finite depth. Institute of Engineering Research, Berkley, 1960 .

Santon (L.). - Enregistrement graphique d'une houle de laboratoire: analyse harmonique. Proc. $V$ Conf. Coastal Engineering, Grenoble, 1952, (188-207).

Stoner (J.J.). - Water waves. Interscience Publishers, N.Y., 1957.

Takano (K.). - C.R.A.S., t. 248, (1768-1771), Paris, 1959; C.R.A.S., t. 249, (1189-1191), Paris, 1959 .

Takano (K.). - Effet d'un obstacle parallélépipédique sur la propagation de la houle. La Houille Blanche, $\mathrm{n}^{\circ} 3$, 1960, (247-267).

TAYLOR (D. G.). - An experimenta study of the transition between oscillatory and solitary waves. M.S. Thesis. Massachussetts Institute of Technology (1955).

URSELL (F.). - The effect of a fixed vertical barrier on surface waves in deep water. Proc. Camb. Phil Soc., $1947,(347-482)$.

VALEmBoIs (J.). - Communication à l'U.G.G.I. Rapport non publié, Rome, 1954.

Wehausen (J. V.). - Surface waves. Handbuch der Physik, Vol. IX. Springer Verlag, 1960.

Wieger (R.L.). - A presentation of cnoïdal wave for practical applications. Journal of Fluid Mechanics, 1960, (273-286). 